\title{
Angiografie ohne Kontrastmittel
}

\section{Siamak Ansari Shahrezaei}

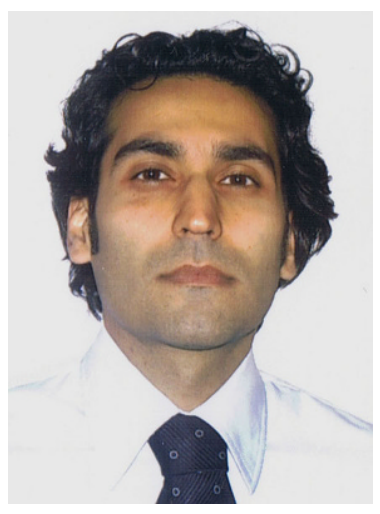

Sehr geehrte Kolleginnen und Kollegen,

durch die rasante Weiterentwicklung der OCT-Technologien und insbesondere der OCT-Angiografie erhalten wir nicht-invasiv eine detaillierte, dreidimensionale Darstellung retinaler Gefäße und Strukturen.
Aus diesem Grund ist die vorliegende Ausgabe des spektrums der augenheilkunde dem Themenschwerpunkt OCT-Angiografie/Medical Retina gewidmet. In unseren Wiener Beiträgen erörtert Dr. Glittenberg die Grundlagen der OCT-Angiografie und stellt eine neue Methode zur choroidalen Gefäßdarstellung vor. Weitere Arbeitsgruppen aus Deutschland, Frankreich, Italien, Kanada, Polen und Schweiz präsentieren ihre Erfahrungen mit diesen neuen Methoden bei verschiedenen retinalen Entitäten.

Den Leserinnen und Lesern wünsche ich vielfältige Anregungen und Wissenszuwachs bei der Lektüre dieser Ausgabe „OCT-Angiografie/Medical Retina“!

Mit kollegialer Hochachtung, Siamak Ansari Shahrezaei

Interessenkonflikt S. Ansari Shahrezaei gibt an, Forschungsunterstützung von Topcon Europe Medical BV erhalten zu haben. 